\title{
Expression analysis of self-incompatibility- associated genes in non-heading Chinese cabbage
}

\author{
L. Wang ${ }^{1,2,3}$, C. Wang ${ }^{1,2,3}$, T.T. Ge ${ }^{1,2,3}$, J.J. Wang ${ }^{1,2,3}$, T.K. Liu ${ }^{1,2,3}$, \\ X.L. Hou ${ }^{1,2,3}$ and Y. $\mathrm{Li}^{1,2,3}$ \\ ${ }^{1}$ Horticultural Department, Nanjing Agricultural University, Nanjing, China \\ ${ }^{2}$ State Key Laboratory of Crop Genetics \& Germplasm Enhancement, \\ Nanjing, China \\ ${ }^{3}$ Key Laboratory of Southern Vegetable Crop Genetic Improvement, \\ Ministry of Agriculture, Nanjing, China \\ Corresponding author: Y. Li \\ E-mail: yingli@njau.edu.cn
}

Genet. Mol. Res. 13 (3): 5025-5035 (2014)

Received June 28, 2013

Accepted November 18, 2013

Published July 4, 2014

DOI http://dx.doi.org/10.4238/2014.July.4.18

\begin{abstract}
In Brassicaceae, a self-incompatibility (SI) system mediates pollen-pistil interactions. Self-pollen could be recognized and rejected by incompatible pistils. Several components involved in the SI response have been determined, including $S$-locus receptor kinase (SRK), $S$-locus cysteine-rich protein/S-locus protein 11, and arm repeatcontaining protein 1 (ARC1). However, the components involved in the SI system of Brassicaceae are not fully understood. Here, we detected expression patterns of 24 SI-related genes in non-heading Chinese cabbage (Brassica campestris ssp chinensis Makino) after compatible and incompatible pollination, and potential interaction relationships of these genes were predicted. $S R K$ and $A R C 1$ transcripts increased initially $0.25 \mathrm{~h}$ after incompatible pollination, while kinase-associated protein phosphatase had an expression pattern that was opposite that of $S R K$ transcripts during self-pollination. Plant U-box 8 was not required in the
\end{abstract}


SI response of non-heading Chinese cabbage. Our results showed that the SI signal of non-heading Chinese cabbage could occur within $0.25 \mathrm{~h}$ after self-pollination. The hypothetical interaction relationships indicated that plastid-lipid-associated protein and malate dehydrogenase could be negatively regulated by chaperonin 10 , glutathione transferase, cytidylate kinase/uridylate kinase, and methionine synthase by indirect interactions. Our findings could be helpful to better understand potential roles of these components in the SI system of non-heading Chinese cabbage.

Key words: Non-heading Chinese cabbage; Pollen germination; Hierarchical clustering; Self-incompatibility; Expression patterns

\section{INTRODUCTION}

Self-incompatibility (SI) is a genetic system that promotes outcross and prevents inbreeding in flowering plants. As a typical sporophytic SI system, Brassicaceae is controlled by a single $S$ locus, which depends on a specific interaction between $S$-locus receptor kinase (SRK) and $S$-locus cysteine-rich protein/S locus protein 11 (SCR/SP11) within the same $S$ haplotype (Ivanov et al., 2010). In addition, some components were also identified to participate in the SI response of Brassicaceae, such as $S$-locus glycoprotein (SLG), arm repeatcontaining protein 1 (ARC1), $M$-locus protein kinase (MLPK), exocyst subunit exo 70 family protein A1 (Exo70A1), plant U-box 8 (PUB8), and kinase-associated protein phosphatase (KAPP) (Vanoosthuyse et al., 2003; Murase et al., 2004; Liu et al., 2007; Samuel et al., 2009).

The rapid SI reaction of Brassicaceae occurs on the stigmatic surface. SLG mediates pollen adhesion, and SRK recognizes SCR/SP11 that is released from the pollen coat (Kachroo et al., 2001; Murase et al., 2004). The activation of receptor-ligand interaction triggers a series of signaling cascades (Kachroo et al., 2001). ARC1 mediates the degradation of Exo70A1 in the SI response through a ubiquitin-mediated degradation pathway (Samuel et al., 2009). However, this ubiquitin-proteasome pathway only presents one branch of the complex SI signaling network (Samuel et al., 2009; Tantikanjana et al., 2010); thus, there could be some other linear pathways involved in the SI response (Tantikanjana et al., 2010; Kitashiba et al., 2011).

To determine the complex SI signaling network, some novel strategies have been performed to explore new candidates involved in the SI system. Samuel et al. (2011) indicated that enolase, tubulin alpha-2/alpha-4 chain, and ribulose-1,5-bisphosphate carboxylase oxygenase (RUBISCO)-large subunit were downregulated after incompatible pollination by two-dimensional gel electrophoresis (2-DE), and they could be related to the SI response of Brassica napus. In addition, cysteine synthase, cytosolic malate dehydrogenase, and ubiquitin-conjugating enzyme were identified to influence SI in apricot and suggested that the SI response could be mediated by cytoskeleton assembly and metabolism processes (Cao et al., 2012). We previously determined that uridine diphosphate (UDP)-sugar pyrophosphorylase and glutathione transferases were involved in the SI response of non-heading Chinese cabbage (Brassica campestris ssp chinensis Makino) by 2-DE (Wang et al., 2014).

To better understand potential roles of incompatible genes related to the SI response, we detected 24 transcripts in pistils of self-compatible and incompatible non-heading Chinese cabbage, including 18 previously identified differentially accumulating genes (Wang et al., 
2014) and six other genes (SRK, SLG, ARC1, MLPK, PUB8, and KAPP). All samples were collected $0,0.25,0.5,1,1.5,2$, and $4 \mathrm{~h}$ after self-pollination at anthesis. Pollen germination on the stigma was also analyzed at the corresponding pollination time. Hierarchical clustering analysis and hypothetical gene interactions were performed. Our findings could be helpful to better understand the potential roles of these genes in the SI system of non-heading Chinese cabbage.

\section{MATERIAL AND METHODS}

\section{Plant materials}

The self-compatible line 210 and incompatible line 002 of non-heading Chinese cabbage (B. campestris ssp chinensis Makino) were grown in Jiangpu Horticultural Experimental Station, Nanjing, China. The 002 is a stable incompatible inbred line after multi-generation selection, and it has a low self-compatibility index $(<1.0)$, whereas that of the 210 line is higher than 5.0. After removing the already opened flowers and siliques, main inflorescences and the neighboring lateral branch were bagged at bud stage. Three to four days later, self-pollination was performed manually on the pistils of freshly opening flowers at anthesis.

\section{Pistil collection}

Self-compatible and incompatible pistils were collected $0.25,0.5,1,1.5,2$, and $4 \mathrm{~h}$ after self-pollination at anthesis and at $0 \mathrm{~h}$ (unpollinated flowers). Triplicate samples were collected per time course, and all samples were cut off and immediately stored in liquid nitrogen.

\section{Pollen germination}

Investigation of self-pollen germination was performed according to the method of Martin (1959). The samples were stored in FAA-fixing solution (formaldehyde:acetic acid:alcohol $=1: 1: 8, \mathrm{v}: \mathrm{v}: \mathrm{v}$ ) for $24 \mathrm{~h}$, washed with ultrapure water, and macerated in $1 \mathrm{~N}$ $\mathrm{NaOH}$ at $45^{\circ} \mathrm{C}$ for $45 \mathrm{~min}$. Finally, the pistils were stained with $0.1 \%$ aniline blue for $12 \mathrm{~h}$. An OLYMPUS BX51 fluorescence microscope was utilized for observations and photographs. Ten pistils of each group $(0,0.25,0.5,1,1.5,2$, and $4 \mathrm{~h})$ were analyzed for manifestation.

\section{Expression patterns of SI-related genes}

Total RNA was extracted from the pistils of compatible and incompatible non-heading Chinese cabbage. The RNAprep Pure Plant Kit (Tiangen, China) was used for RNA extraction, and PrimeScript RT Reagent Kit (TaKaRa, China) was used to generate cDNA. The Beacon Designer v7.51 (Premier Biosoft International, Palo Alto, CA, USA) was used to design specific primers of the 24 genes. Actin was chosen as the reference gene to normalize expression levels between different samples. All gene-specific primers were listed in Table S1.

SYBR Premix Ex Taq (Tli RNaseH Plus; TaKaRa) was utilized, and a $20-\mu \mathrm{L}$ reaction mixture was added to each well. The polymerase chain reaction (PCR) was performed at $95^{\circ} \mathrm{C}$ for $30 \mathrm{~s}$ and 40 cycles of $95^{\circ} \mathrm{C}$ for $3 \mathrm{~s}$ and $60^{\circ} \mathrm{C}$ for $30 \mathrm{~s}$ on a 7500 Fast Real-Time PCR System (Applied Biosystems, Foster City, CA, USA). The relative mRNA abundance was calculated 
from the threshold cycle $(\mathrm{Ct})$, and gene expression was evaluated by the relative quantification $\left(\Delta \Delta C_{t}\right)$ (Livak and Schmittgen, 2001). The standard deviation (SD) was calculated. The reactions were run for three replicates of each sample.

\section{Hierarchical clustering analysis and data visualization}

Gene expression data were input into Cluster 3.0, and the average-linkage method was chosen (de Hoon et al., 2004). Visualization of gene expression profiling was performed with Java Treeview (Saldanha, 2004). Annotations of the 24 genes were obtained from the Gene Ontology (GO) database (http://www.geneontology.org/).

\section{Development of an interaction gene network among SI-related genes}

Because a web-based network analytical tool for B. campestris was not established, and given that Arabidopsis and non-heading Chinese cabbage both belong to Cruciferae, we used a web-tool that was developed for Arabidopsis. Arabidopsis interaction viewer (http:// bar.utoronto.ca/interactions/cgi-bin/arabidopsis_interactions_viewer.cgi) is a powerful tool for developing hypothetical functional gene networks (Geisler-Lee et al., 2007). The $24 \mathrm{Ara}-$ bidopsis orthologs were queried in Arabidopsis interaction viewer, and the following parameters were selected: filter output to remove duplicate interactions, show only interactions from published data sets, and include additional protein information from MapMan. The output data were analyzed to predict hypothetical gene interactions.

\section{RESULTS}

\section{Pollen growth after self-pollination at anthesis}

Differential pollen growths of pollen grains were observed on the compatible and incompatible stigmatic surface of non-heading Chinese cabbage. Self-pollen grains could rapidly adhere and hydrate on the compatible stigma epidermal cell at $0.5 \mathrm{~h}$ (Figure $1 \mathrm{~A}-\mathrm{C}$ ). Then, the pollen germinated and produced pollen tubes to penetrate the stigmatic surface $1 \mathrm{~h}$ after self-pollination (Figure 1D); the pollen tubes continued to elongate to the style for the next 3 $\mathrm{h}$ (Figure 1E-G). In contrast, we found that a limited number of pollen grains could adhere on the incompatible stigmatic surface within $0.25 \mathrm{~h}$ (Figure $1 \mathrm{H}$ and I), but most rejected pollen grains failed to attach and were washed away during the aniline blue staining. Furthermore, these adhered pollen grains displayed poor germination, and few pollen tubes were produced during 0.5 to $1 \mathrm{~h}$ after incompatible pollination (Figure $1 \mathrm{~J}$ and $\mathrm{K}$ ). Abundant callose was also observed in the incompatible stigma epidermal cell, which could block pollen tubes from penetrating the stigmatic surface (Figure 1L). The incompatible pollination resulted in stunted pollen tubes, which appeared to stop growing $4 \mathrm{~h}$ after self-pollination (Figure $1 \mathrm{M}$ and $\mathrm{N}$ ).

The statistics on germinated pollen grain number and pollen tube length were also performed after self-pollination. Approximately seven germinated pollen grains were detected $0.25 \mathrm{~h}$ after incompatible pollination, whereas 33 pollen grains were found to germinate on compatible stigmatic surface (Figure 2A). Similarly, the length of compatible pollen tubes was 9.2-fold greater than that of incompatible ones $0.25 \mathrm{~h}$ after self-pollination (Figure 2B). Fur- 
thermore, we found that compatible pollen tubes grew rapidly after $1.5 \mathrm{~h}$ through self-pollination, whereas the growth rate of incompatible pollen tubes remained unchanged (Figure 2B).

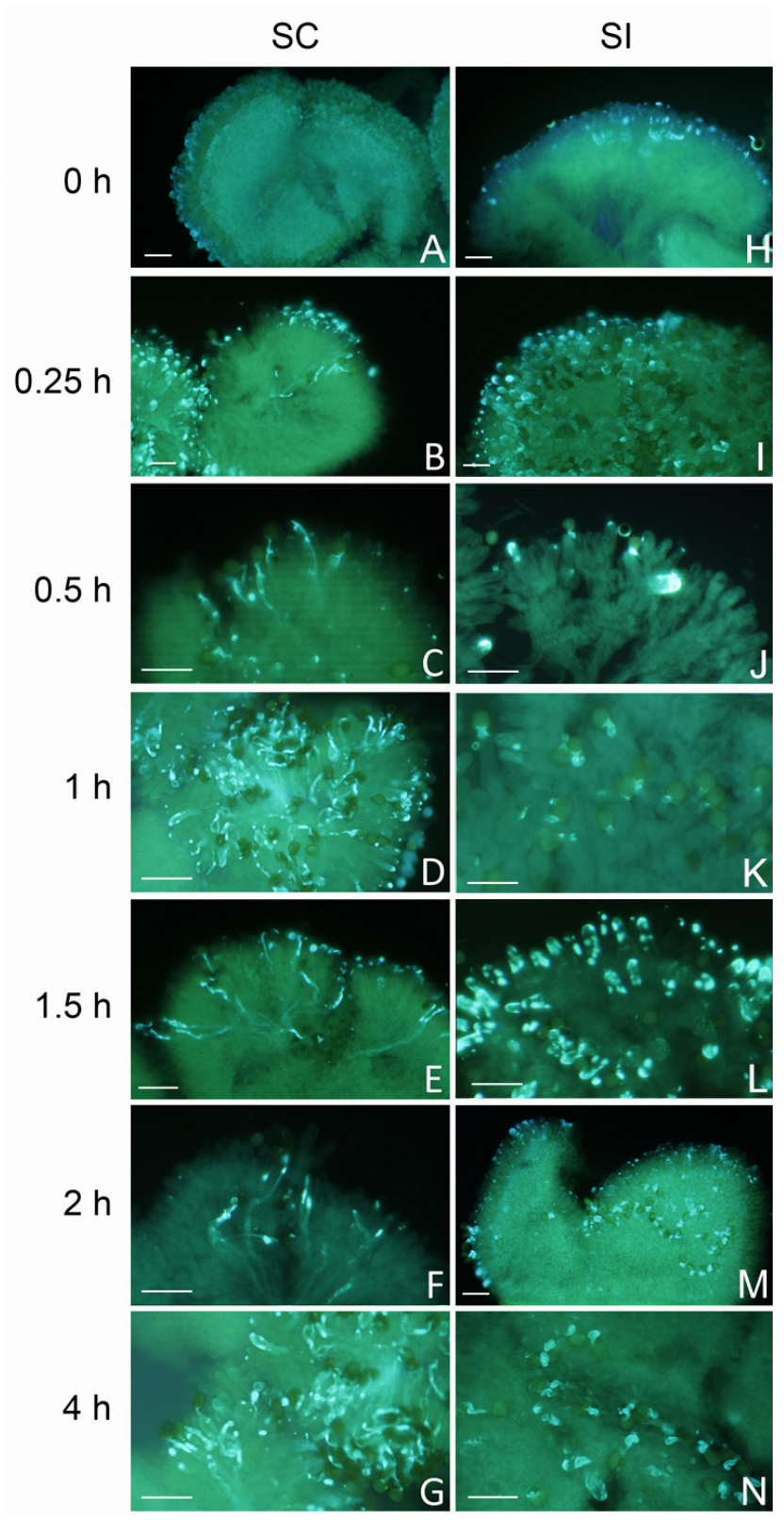

Figure 1. Pollen germination and pollen tube growth on the self compatible and incompatible stigma. (A) to (N) show pollen adhesion, germination and pollen tube growth at $0,0.25,0.5,1,1.5,2$, and $4 \mathrm{~h}$ after self-pollination, respectively. SC and SI indicate the self-compatible and -incompatible stigma, respectively. Bars $=100 \mu \mathrm{m}$. 

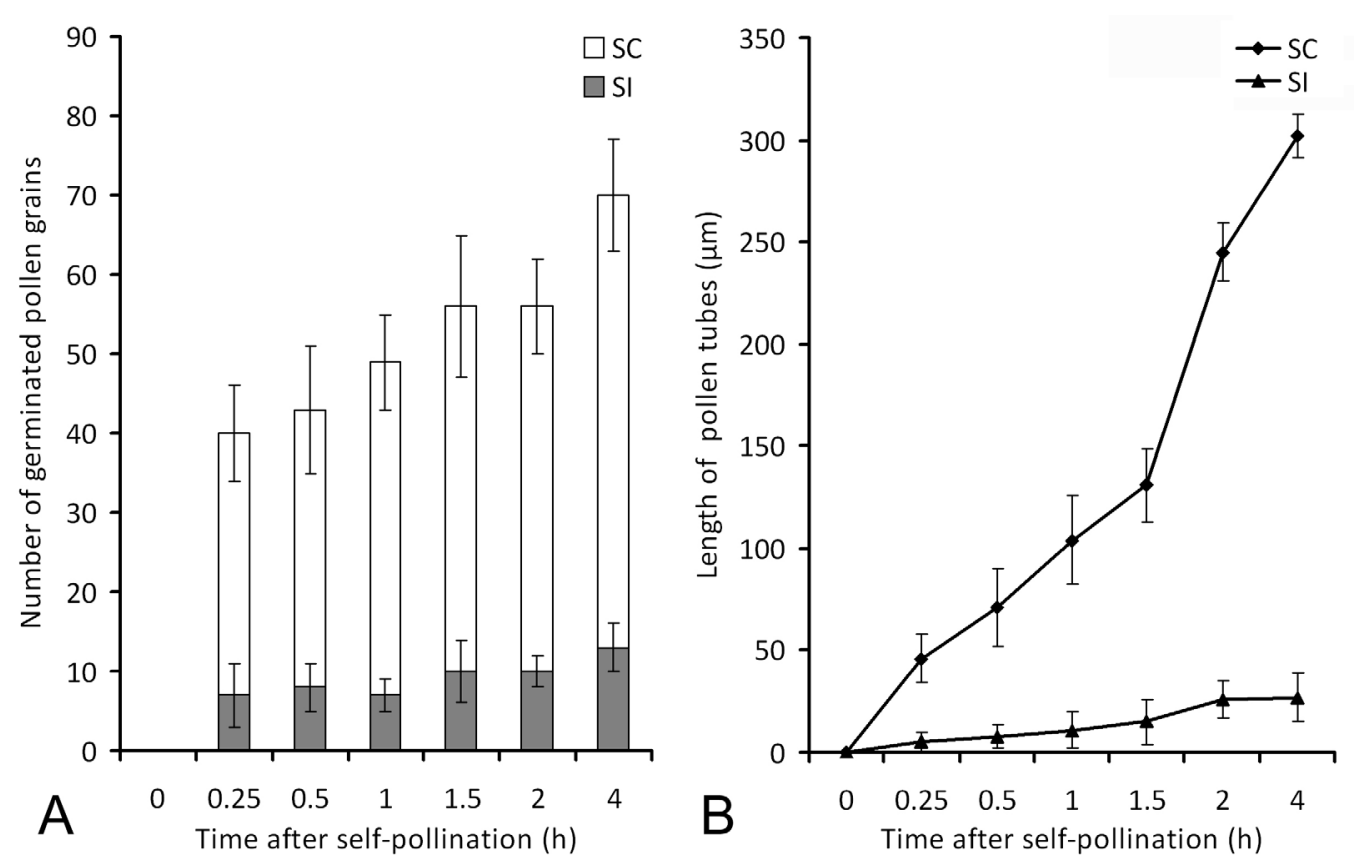

Figure 2. Pollen germination number and pollen tube growth on the self-compatible and -incompatible stigma. A. Pollen germination was detected by counting average number of germinated pollen grains at $0.25,0.5,1,1.5,2$, and $4 \mathrm{~h}$ after self-pollination. White and gray bars indicate the number of germinated pollen after self-compatible (SC) and -incompatible (SI) pollination, respectively. B. Pollen tube growth was detected by measuring length of pollen tubes. Lozenges and triangles indicate length of pollen tubes after SC and SI pollination, respectively. $\mathrm{N}=$ 10. Error bars are \pm SE.

\section{Hierarchical clustering analysis of SI-related genes}

Twenty-four transcripts in self-compatible and -incompatible pistils of non-heading Chinese cabbage were detected $0,0.25,0.5,1,1.5,2$, and $4 \mathrm{~h}$ after self-pollination at anthesis. The expression data were log-transformed and performed with Java Treeview (Figure 3).

We found that seven genes, KAPP, SLG, PUB8, senescence-associated cysteine protease $(C P)$, Bet $\mathrm{v} 1$ allergen family protein $(B e t v 1)$, proteasome $20 \mathrm{~S}$ beta1, and pollen-specific protein Bnm1, were co-expressed and reduced within the initial $0.25 \mathrm{~h}$ of pollination, and then they were upregulated approximately $0.5 \mathrm{~h}$ after compatible pollination. A total of nine genes, including $M L P K, A R C 1$, rubisco ssu precursor, cytidylate kinase/uridylate kinase (PYRO), Rieske FeS protein, strictosidine synthase, malate dehydrogenase $(M D H)$, glutathione S-transferase, and glutathione transferase, showed an upregulated transcriptional tendency in self-compatible plants within the initial 0.25 or $0.5 \mathrm{~h}$, and expression declined $1 \mathrm{~h}$ after self-pollination (Figure 3A). Four genes, including aconitase C-terminal domain-containing protein $(A C D C)$, nicotinamide adenine dinucleotide phosphate-dependent glyceraldehyde3-phosphate dehydrogenase (NADP-GAPDH), UDP-sugar pyrophosphorylase (USP), and plastid-lipid-associated protein (PAP2), displayed a continuous upregulation until $4 \mathrm{~h}$ after compatible pollination. The other four genes, SRK, chaperonin 10 (CPN10), methionine syn- 


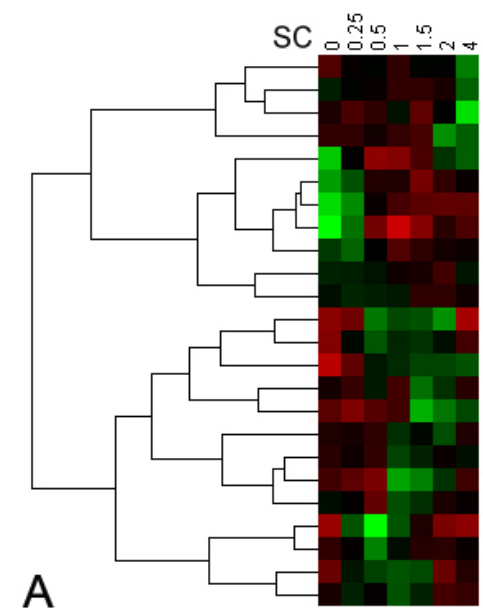

Aconitase C-terminal domain-containing protein, $A C D C$

NADP-dependent glyceraldehyde-3-phosphate dehydrogenase, NADP-GAPDH

UDP-sugar pyrophosphorylase, USP

Plastid-lipid-associated protein PAP2

Kinase-associated protein phosphatase, KAPP

S-locus glycoprotein, SLO

Plant U-box 8, PUB8

Senescence-associated cysteine protease, $\mathrm{CP}$

Bet $v 1$ allergen family protein

Proteasome $20 \mathrm{~S}$ beta 1

Pollen-specific protein Bnm1

M-locus protein kinase, MLPK

Rubisco ssu precursor

Cytidylate kinasejuridylate kinase, PYR6

Rieske FeS protein

Strictosidine synthase

Arm repeat containing protein $1, \mathrm{ARC} 1$

Malate dehydrogenase, $\mathrm{MDH}$

Glutathione S-transferase

Glutathione transferase

S-locus receptor kinase, SRK

Chaperonin 10, CPN10

Methionine synthase, MS

Aspartic protease. AP

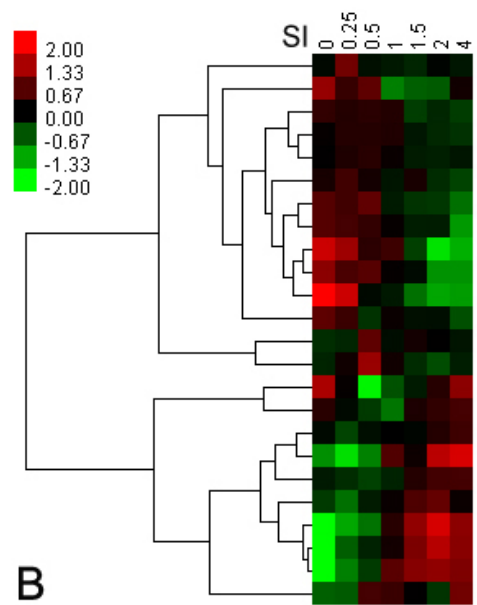

S-locus glycoprotein, SLO

Malate dehydrogenase, $\mathrm{MDH}$

Arm repeat containing protein $1, \mathrm{ARC} 1$

S-locus receptor kinase, SRK

Glutathione S-transferase

M-locus protein kinase, MLPK

NADP-dependent glyceraldehyde-3-phosphate dehydrogenase, NADP-GAPDH

Rieske Fes protein

Plastid-lipid-associated protein PAP2

Strictosidine synthase

Pollen-specific protein Bnm1

Rubisco ssu precursor

UDP-sugar pyrophosphorylase, USP

Methionine synthase, MS

Kinase-associated protein phosphatase, KAPP

Aspartic protease, AP

Aspartic protease, AP
Plant U-box 8, PUB8

Plant U-bo 8 , PUB8

Aconitase $\mathrm{C}$-terminal domain
Bet 1 allergen family protein

Cytidylate kinasejuridylate kinase, PYR

Proteasome $20 \mathrm{~S}$ beta 1

Senescence-associated cysteine protease, $\mathrm{CP}$

Chaperonin 10, CPN10

Glutathione transferase

Figure 3. Cluster analysis of self-incompatible-related genes after compatible and incompatible pollination. The cluster analysis utilized the expression data of 24 genes at $0,0.25,0.5,1,1.5,2$, and $4 \mathrm{~h}$ after self-pollination. A. Clustered expression data after self-compatible (SC) pollination. B. Clustered data after self-incompatible (SI) pollination. Green, red and dark blocks indicate downregulation, upregulation and no change in mRNA levels, respectively. Green and red bars indicate downregulation and upregulation at $0.25 \mathrm{~h}$ after self-pollination, respectively.

thase $(M S)$, and aspartic protease $(A P)$, had reduced expression at $0.25 \mathrm{~h}$ that increased $1.5 \mathrm{~h}$ after compatible pollination (Figure $3 \mathrm{~A}$ ).

Differential transcriptional levels of the 24 genes were present in self-incompatible plants. Eight genes, PUB8, ACDC, Bet v1, PYR6, proteasome 20S beta1, CP, CPN10, and glutathione transferase, showed a similar transcriptional tendency and were downregulated at 0.25 or $0.5 \mathrm{~h}$ and upregulated $1 \mathrm{~h}$ after incompatible pollination. Twelve genes, $S L G, M D H$, $A R C 1, S R K$, glutathione S-transferase, $M L P K, N A D P-G A P D H$, Rieske FeS protein, PAP2, strictosidine synthase, Bnml, and rubisco ssu precursor, had increased expression within the initial 0.25 or $0.5 \mathrm{~h}$ that declined $1 \mathrm{~h}$ after self-pollination (Figure 3B). In addition, USP and $M S$ were upregulated at $0.5 \mathrm{~h}$ and downregulated $1 \mathrm{~h}$ after incompatible pollination, while 
$K A P P$ and $A P$ were downregulated at $0.5 \mathrm{~h}$ and upregulated $1.5 \mathrm{~h}$ after incompatible pollination (Figure $3 \mathrm{~B}$ ).

A hierarchical clustering analysis was performed by combining the expression data of compatible and incompatible plants after self-pollination. The self-pollination process was divided into two groups. Group A consisted of the data of self-incompatible pollination ( 0 to 4 h) and self-compatible pollination $(0$ and $0.25 \mathrm{~h})$, and group B contained the rest of the data of self-compatible pollination $(0.5$ to $4 \mathrm{~h}$ ) (Figure 4$)$. In the self-incompatible pollination process, the data from 0 and $0.25 \mathrm{~h}$ were clustered in a subgroup, and the other five data points $(0.5,1$, $1.5,2$, and $4 \mathrm{~h}$ ) belonged to another subgroup. Similarly, the self-compatible $0.25 \mathrm{~h}$ data were different from the other five data points $(0.5,1,1.5,2$, and $4 \mathrm{~h})$ (Figure 4$)$.

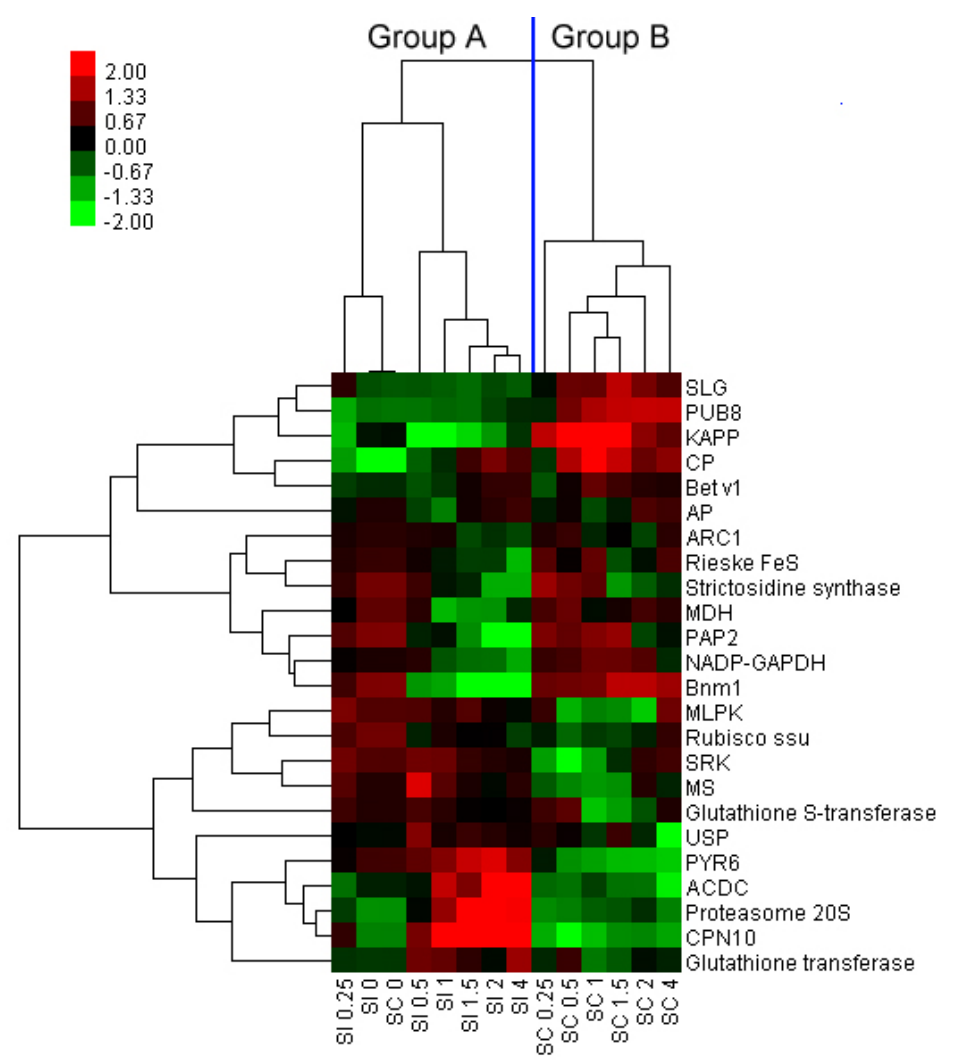

Figure 4. Cluster analysis of expression data combined with compatible and incompatible pollination. The time course combined $0,0.25,0.5,1,1.5,2$, and $4 \mathrm{~h}$ following compatible and incompatible pollination. Rows represent expression levels of 24 genes at $0,0.25,0.5,1,1.5,2$, and $4 \mathrm{~h}$ after self-pollination, and columns represent selfpollination time course.

\section{Hypothetical gene interactions of SI-related genes}

Using the Arabidopsis interaction viewer, interaction relationships between these 24 SI-related genes were investigated (Figure 5 and Table S2). Based on GO annotations, 
six genes related to energy metabolism and stress response, including PAP2, CPN10, MDH, glutathione transferase, PYR6, and MS, constituted a hypothetical interaction network, and indirect interactions could occur between these six genes through links of RNA polymerase I subunit 43 (At1g60620), 6-phosphogluconate dehydrogenase family protein (At1g64190), heat-shock protein 90.1 (At5g52640), P-loop containing nucleoside triphosphate hydrolase superfamily protein (At5g59440), RING/U-box superfamily protein (At1g65040), and importin alpha isoform 6 (At1g02690), respectively (Figure 5).

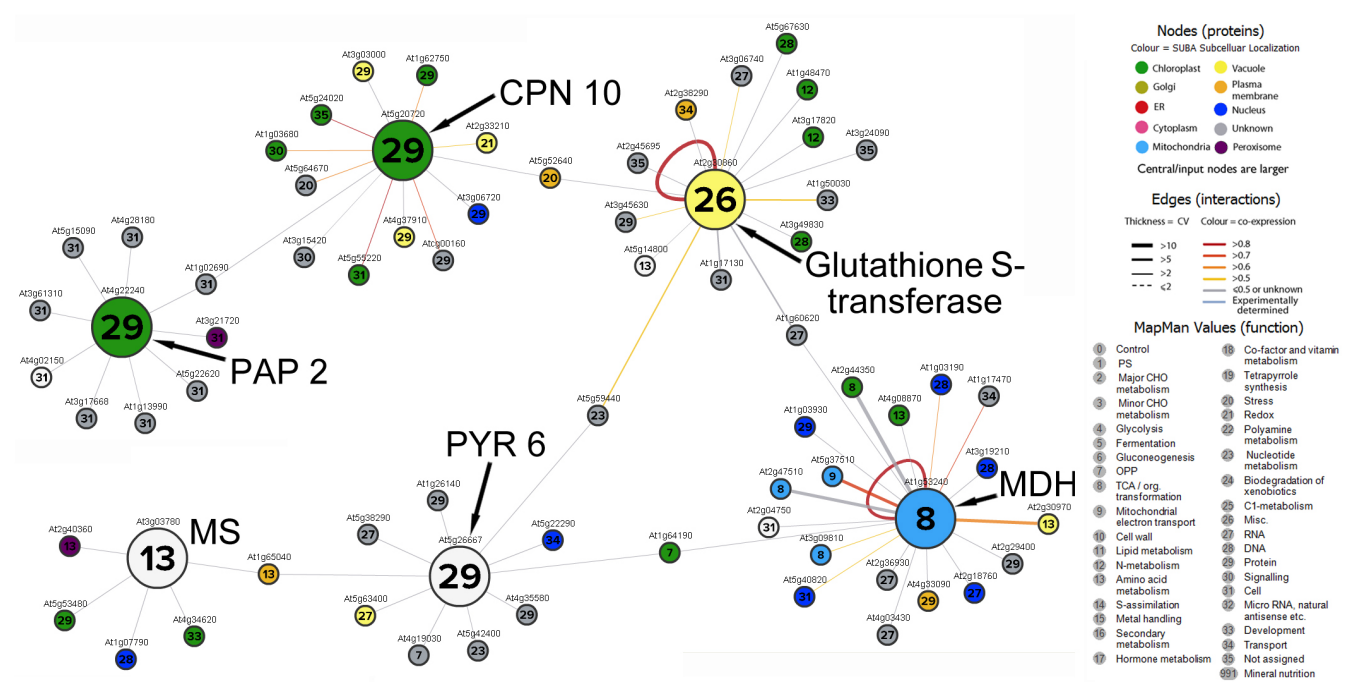

Figure 5. Interaction network of part SI-related genes. A node indicates a protein, and mapman value in each node indicates function of the protein. The color of each node indicates different subcellular localizations (in upper right panel), and edges indicate interaction strength.

\section{DISCUSSION}

The SI response consists of a series of signaling cascades, including protein phosphorylation, dephosphorylation, ubiquitination, and degradation (Tantikanjana et al., 2010). The SI signal is activated to prevent self-pollen hydration, germination, and pollen tube production on the stigmatic surface in a short time (Ivanov and Gaude, 2009). Samuel et al. (2009) found that incompatible pollen grains of B. napus $\mathrm{W} 1$ plants displayed poor hydration and slow increase within 10 min following self-pollination. We observed similar pollen germination at $0.25 \mathrm{~h}$ on the stigma of self-incompatible non-heading Chinese cabbage. Compared with the 33 germinated pollen grains on the compatible stigma, only seven pollen grains germinated at $0.25 \mathrm{~h}$ and produced stunted pollen tubes after incompatible pollination (Figure 2). Thus, we hypothesized that the SI signal occurred within the initial $0.25 \mathrm{~h}$ after incompatible pollination in non-heading Chinese cabbage.

The expression patterns of 24 SI-related genes also supported this hypothesis. Results revealed that most genes displayed an opposite expression tendency between compatible and incompatible plants $0.25 \mathrm{~h}$ after self-pollination (Figures 3 and 4). Among them, SRK was the female $S$-determinant in the SI system, which reached the highest levels on the day of anthesis and remained highly expressed in the early stages after self-pollination (Liu et al., 2007). In our study, the $S R K$ expression level increased in the initial $0.25 \mathrm{~h}$ and reached a peak $1 \mathrm{~h}$ 
after incompatible pollination, along with the upregulation of $S L G$ (Figure 3B). The mRNA level of $K A P P$ was opposite that of the $S R K$ transcript during self-pollination in non-heading Chinese cabbage. This could be because of the negative regulator role of KAPP in the SI response, which mediated the downregulation of SRK by dephosphorylation (Williams et al., 1997; Vanoosthuyse et al., 2003). ARC1 displayed a similar transcriptional tendency as $S R K$ in self-incompatible non-heading Chinese cabbage after self-pollination because ARC1 was phosphorylated by SRK in the Brassicaceae SI system (Gu et al., 1998). Our results showed that the CPN10 transcript increased after incompatible pollination, which played an important role in the stress response (Wang et al., 2004). Poulter et al. (2011) showed that chaperonins were only found in self-incompatible Papaver after pollination. This implied that the SI response triggered a stress response and induced chaperonins abundance in self-incompatible non-heading Chinese cabbage. Liu et al. (2007) indicated that PUB8 was required to maintain sufficient SRK mRNA levels in the SI response of transgenic Arabidopsis thaliana. They revealed that PUB8 represented a novel U-box/ARM repeat protein, which is a functional U-box E3 ligase that is required for SI in Arabidopsis. However, we found that PUB8 displayed no difference in expression levels between self-compatible and incompatible non-heading Chinese cabbage after self-pollination. Thus, PUB8 might not be required in the SI response of non-heading Chinese cabbage because ARC1, also an E3 ubiquitin ligase, was involved in the ubiquitin-mediated degradation pathway in the SI response.

We found a hypothetical gene interaction network that consisted of PAP2, CPN10, $M D H$, glutathione transferase, $P Y R 6$, and $M S$, which implied that energy metabolism and stress response would be involved in self-pollen recognition and rejection (Poulter et al., 2011; Cao et al., 2012). Among them, CPN10, glutathione transferase, $P Y R 6$, and $M S$ were upregulated in self-incompatible plants after self-pollination, while $P A P 2$ and $M D H$ were downregulated. This suggested that $P A P 2$ and $M D H$ might be negatively regulated in the SI response. The interaction network showed that $P A P 2$ was negatively induced by $C P N 10$ through the link of importin alpha isoform 6 (At1g02690), which could rapidly accumulate in the nucleus in response to stress (Yasuda et al., 2012). MDH was negatively induced by glutathione transferase and PYR6 through links of RNA polymerase I subunit 43 (At1g60620) and 6-phosphogluconate dehydrogenase family protein (At1g64190), respectively. Tantikanjana et al. (2009) determined that RNA-dependent RNA polymerase (RDR6) could enhance the SI response of transgenic self-incompatible $A$. thaliana. It is known that $A$. thaliana is self-compatible; thus, our prediction based on Arabidopsis interaction data could predict hypothetical gene interactions in the SI response of non-heading Chinese cabbage, and further research is required to examine their relationships.

Based on the analysis of expression patterns of 24 genes in self-compatible and incompatible non-heading Chinese cabbage $0,0.25,0.5,1,1.5,2$, and $4 \mathrm{~h}$ after self-pollination, we concluded a relevance between the pollen growth process and gene expression tendency as follows: 1) the SI signal of non-heading Chinese cabbage could occur within the initial $0.25 \mathrm{~h}$ after self-pollination; 2) PUB8 might not be required in the SI response of non-heading Chinese cabbage; and 3) PAP2 and MDH could be negatively regulated by CPN10, glutathione transferase, and PYR6 through indirect interactions. Our findings could provide a better understanding of these gene functions in the SI system of non-heading Chinese cabbage.

\section{ACKNOWLEDGMENTS}

Research supported by the National Basic Research Program of China ("973" 
Program, \#2009CB119001), the Fundamental Research Funds for the Central Universities of China (\#KYZ201111), the Natural Science Foundation of Jiangsu Province (\#BK2011643), the Specialized Research Fund for the Doctoral Program of Higher Education (SRFDP, \#BO201300666), and the Science and Technology Pillar Program of Jiangsu Province (\#BE2012325).

\section{Supplementary material}

\section{REFERENCES}

Cao X, Feng J, Wang D, Sun J, et al. (2012). Primary style protein expression in the self-incompatible/compatible apricot by the 2D-DIGE technique. Gene 503: 110-117.

de Hoon MJ, Imoto S, Nolan J and Miyano S (2004). Open source clustering software. Bioinformatics 20: 1453-1454.

Geisler-Lee J, O’Toole N, Ammar R, Provart NJ, et al. (2007). A predicted interactome for Arabidopsis. Plant Physiol. 145: 317-329.

Gu T, Mazzurco M, Sulaman W, Matias DD, et al. (1998). Binding of an arm repeat protein to the kinase domain of the S-locus receptor kinase. Proc. Natl. Acad. Sci. U. S. A. 95: 382-387.

Ivanov R and Gaude T (2009). Endocytosis and endosomal regulation of the S-receptor kinase during the selfincompatibility response in Brassica oleracea. Plant Cell 21: 2107-2117.

Ivanov R, Fobis-Loisy I and Gaude T (2010). When no means no: guide to Brassicaceae self-incompatibility. Trends Plant Sci. 15: 387-394.

Kachroo A, Schopfer CR, Nasrallah ME and Nasrallah JB (2001). Allele-specific receptor-ligand interactions in Brassica self-incompatibility. Science 293: 1824-1826.

Kitashiba H, Liu P, Nishio T, Nasrallah JB, et al. (2011). Functional test of Brassica self-incompatibility modifiers in Arabidopsis thaliana. Proc. Natl. Acad. Sci. U. S. A. 108: 18173-18178.

Liu P, Sherman-Broyles S, Nasrallah ME and Nasrallah JB (2007). A cryptic modifier causing transient self-incompatibility in Arabidopsis thaliana. Curr. Biol. 17: 734-740.

Livak KJ and Schmittgen TD (2001). Analysis of relative gene expression data using real-time quantitative PCR and the 2(-Delta Delta C(T)) method. Methods 25: 402-408.

Martin FW (1959). Staining and observing pollen tubes in the style by means of fluorescence. Stain Technol. 34: 125-128.

Murase K, Shiba H, Iwano M, Che FS, et al. (2004). A membrane-anchored protein kinase involved in Brassica selfincompatibility signaling. Science 303: 1516-1519.

Poulter NS, Bosch M and Franklin-Tong VE (2011). Proteins implicated in mediating self-incompatibility-induced alterations to the actin cytoskeleton of Papaver pollen. Ann. Bot. 108: 659-675.

Saldanha AJ (2004). Java Treeview - extensible visualization of microarray data. Bioinformatics 20: 3246-3248.

Samuel MA, Chong YT, Haasen KE, Aldea-Brydges MG, et al. (2009). Cellular pathways regulating responses to compatible and self-incompatible pollen in Brassica and Arabidopsis stigmas intersect at Exo70A1, a putative component of the exocyst complex. Plant Cell 21: 2655-2671.

Samuel MA, Tang W, Jamshed M, Northey J, et al. (2011). Proteomic analysis of Brassica stigmatic proteins following the selfincompatibility reaction reveals a role for microtubule dynamics during pollen responses. Mol. Cell Proteomics 10: M111.

Tantikanjana T, Rizvi N, Nasrallah ME and Nasrallah JB (2009). A dual role for the S-locus receptor kinase in selfincompatibility and pistil development revealed by an Arabidopsis rdr6 mutation. Plant Cell 21: 2642-2654 .

Tantikanjana T, Nasrallah ME and Nasrallah JB (2010). Complex networks of self-incompatibility signaling in the Brassicaceae. Curr. Opin. Plant Biol. 13: 520-526.

Vanoosthuyse V, Tichtinsky G, Dumas C, Gaude T, et al. (2003). Interaction of calmodulin, a sorting nexin and kinaseassociated protein phosphatase with the Brassica oleracea $S$ locus receptor kinase. Plant Physiol. 133: 919-929.

Wang L, Peng H, Ge T, Liu T, et al. (2014). Identification of differentially accumulating pistil proteins associated with self-incompatibility of non-heading Chinese cabbage. Plant Biol. 16: 49-57.

Wang W, Vinocur B, Shoseyov O and Altman A (2004). Role of plant heat-shock proteins and molecular chaperones in the abiotic stress response. Trends Plant Sci. 9: 244-252.

Williams RW, Wilson JM and Meyerowitz EM (1997). A possible role for kinase-associated protein phosphatase in the Arabidopsis CLAVATA1 signaling pathway. Proc. Natl. Acad. Sci. U. S. A. 94: 10467-10472.

Yasuda Y, Miyamoto Y, Yamashiro T, Asally M, et al. (2012). Nuclear retention of importin alpha coordinates cell fate through changes in gene expression. EMBO J. 31: 83-94. 